\title{
Performance Analysis of Multiband Impulse Radio UWB Communication System Based on PSWF
}

\author{
Lili Chen, Zheng Dou \\ Information and Communication Engineering College, Harbin Engineering University, Harbin, China \\ Email: douzheng@hrbeu.edu.cn
}

Received May, 2013

\begin{abstract}
To improve the data transmission rate and use spectrum flexibly, a new spectrum allocation method for Multiband Impulse Radio UWB (MB-IR-UWB) is proposed in this paper based on the band-limited and orthogonal characteristics of Prolate Spheroidal Wave Function (PSWF). The system model is built and the bit error rate (BER) formula is deprived by binary time hopping pulse position modulation under additive white Gaussian noise. Moreover, the system performance is analyzed via MATLB simulation. The results indicate that MB-IR-UWB system performance of BER is the same with single-band UWB. However, in the proposed scheme the data can be transmitted in multiple parallel bands, which enjoys much higher transmission rate. In addition, PSWF pulse duration affects the BER performance.
\end{abstract}

Keywords: IR-UWB; Multiband; PSWF; Performance Analysis

\section{Introduction}

Ultra-wideband (UWB) is an emerging technology that offers great promises to satisfy the growing demand for low cost and high-speed digital wireless home networks. By using ultra short impulse pulse to convey information [1], Impulse Radio allows remarkable data rates of Gbit/s. However, conventional single-band UWB signal has a wide fixed spectrum, which made the receiver lack the flexibility of spectrum utilization and restricted the improvement of the system transmission performance. In 2004, Stkphane Paquelet proposed the idea of multiband in $[2,3]$, and proved that the method can effectively improve the transmission rate. Later in [4] Martin Mittelbach modified the model and concluded that extending the basic architecture to a multiband system could allow remarkable data rates in the order of several hundred Mbit/s up to almost a Gbit/s. Multiband Impulse Radio UWB (MB-IR-UWB) scheme divides the allocated UWB frequency range into several subbands and transmit information in multiple parallel band, which would not only expand the capacity of the system, but also improve the flexibility of spectrum utilization. By using soft-spectrum adaption (SSA) [5], it can avoid some certain narrow-band interference and improve the performance of receiver. Therefore, this scheme will become a new direction of future technologies.

According to preceding researches, we know traditional MB-IR-UWB systems use band-pass filter to arrange for the spectrum, and use incoherent energy detection in the receiver, but the filter design is more complex.
Based on the band-limited and orthogonal characteristics of PSWF, it can be equivalent to the ideal band-pass filter, and the coherent demodulation can be applied to get better performance.

In this paper, Section II makes a brief introduction of MB-IR-UWB architecture. The detailed description of waveform choice, band allocation, and system model is developed in Section III. The simulation result and analysis of transmission performance based on PSWF is presented and discussed in Section IV. Finally, Section V concludes this paper.

\section{MB-IR-UWB System Overview}

A block diagram of entire system model is depicted in Figures 1 and 2. These two diagrams contain the major components of this architecture [6,7].

The basic working method of MB-IR-UWB system is as follows. A mono-band pulse generator is to generate a number of pulses where each pulse occupies a specific frequency band. The relatively narrow-band pulses are referred to as subband or monoband pulses. Each subband pulse is modulated with different data according to a specific modulation scheme. Prior to modulation the coded bit stream is convert into a block of $M N_{\text {band }}$ bits that is partitioned into $N_{\text {band }}$ groups of $M$ bits. After modulation, this sum of subband pulses forms the MBIR-UWB transmit signal and is transmitted via the single UWB antenna. After experiencing a multi-path and thermal noise channel, on the receiver side, a coherent 
demodulation is considered, ensuring the optimum reception. At first, the received multi-band signal is decomposed by multiplying the orthogonal mono-band pulse to its subbands and subsequent operations are performed per band. And then, make the received data parallel to serial conversion and make a soft or hard bit decision depending on the type of channel decoder. At last, we can get the original data. Theoretically multi-band technology can improve the system capacity $N$ times ( $N$ is the sub-band number), but it is still far from this limit.

\section{MB-IR-UWB System Model Building}

\subsection{Prolate Spheroidal Wave Function Set}

Prolate Spheroidal Wave Function (PSWF) has the advantage of time-limited and band-limited. It was first proposed by D.Slepian and H.O.Pollack of Bell Lab in the late 1950s [8]. It is a complete set of orthogonal function, which is time-limited in $[-T / 2, T / 2]$ and bandlimited in $[-\Omega, \Omega]$. It has the maximum energy concentration on the interval $[-T, T]$ as a band limited function. The PSWF pulses are orthogonal both in time and frequency domain. We can shift its spectrum and control its bandwidth easily by adjusting the pulse parameters. Therefore it is often applied in UWB communication.

As is well-known, it's difficult to calculate the closedform solution of PSWF. So the discrete approximation solving method has been used, which can be regarded as follows. The signal $\phi(t)$, whose time duration is $T$, goes through an ideal band-pass filter that the upper cut-off

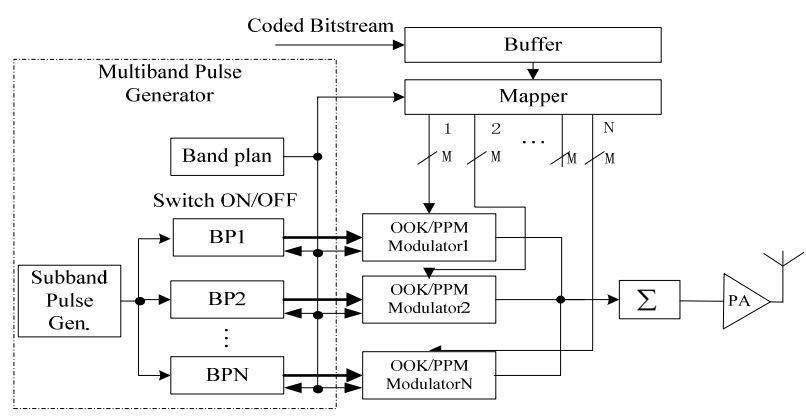

Figure 1. Multi-band pulse generator and MB-IR-UWB transmitter.

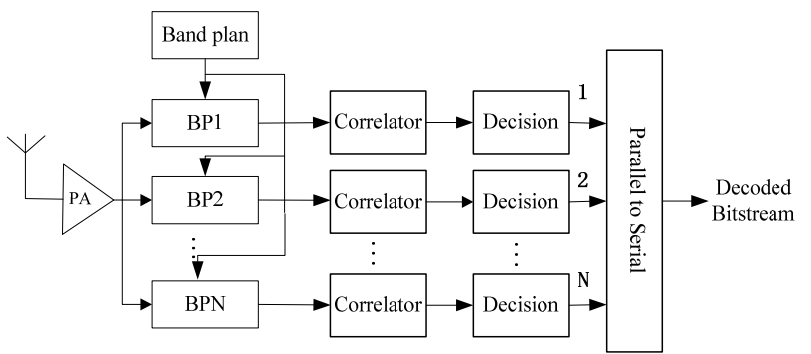

Figure 2. MB-IR-UWB receiver block diagram. frequency is $f_{u}$ and lower cut-off frequency is $f_{l}$, the output signal is $\lambda \phi(t)$. So the $N$ base frequency bands can be regarded as $N$ ideal band-pass filter. The template of $N$ spectrum is as follows.

$$
\begin{aligned}
& h_{i}(t)=2 f_{i, u} \sin c\left(2 f_{i, u} t\right)-2 f_{i, l} \sin c\left(2 f_{i, l} t\right) \\
& H(f)=\left\{\begin{array}{lc}
1 & f_{i, l}<f_{i}<f_{i, u} \\
0 & \text { others }
\end{array} \quad i \in(1, N)\right.
\end{aligned}
$$

where $f_{i, l}$ and $f_{i, u}$ respectively means the lower cutoff frequency and upper cut-off frequency of the ith base frequency band. Using discrete solution respectively in each band, the corresponding PSWF pulses of all orders can be attained. In this paper, we choose the first order PSWF pulses of each band as the base pulses.

For two band-limited PSWF pulses $\phi_{i, k}(t)$ and $\phi_{j, l}(t)$, in different sub-bands, they don't overlap each other in the frequency domain. By the Parseval's theorem, it can be easily proved that

$$
\int_{-/ / 2}^{T / 2} \phi_{i, k}(t) \phi_{j, l}(t) d t=\delta_{i, j}= \begin{cases}1 & i=j \\ 0 & i \neq j\end{cases}
$$

So the band pass PSWFs in different sub-bands are orthogonal [9]. Due to the double orthogonal characteristic, two PSWF pulses have the same time duration and subbands are orthogonal. Therefore, all the pulses in the set are mutually orthogonal.

In this paper, we divide the UWB spectrum (from 3.1 $\mathrm{GHz}$ to $7.5 \mathrm{GHz}$ ) into several subbands, and then use the corresponding PSWF time domain impulse as the information carrier of MB-IR-UWB. The multiband spectrum allocation is given in Figure 3. In order to meet the requirements of ultra-wideband communications, the bandwidth of each band should be greater than or equal to $500 \mathrm{MHz}$.

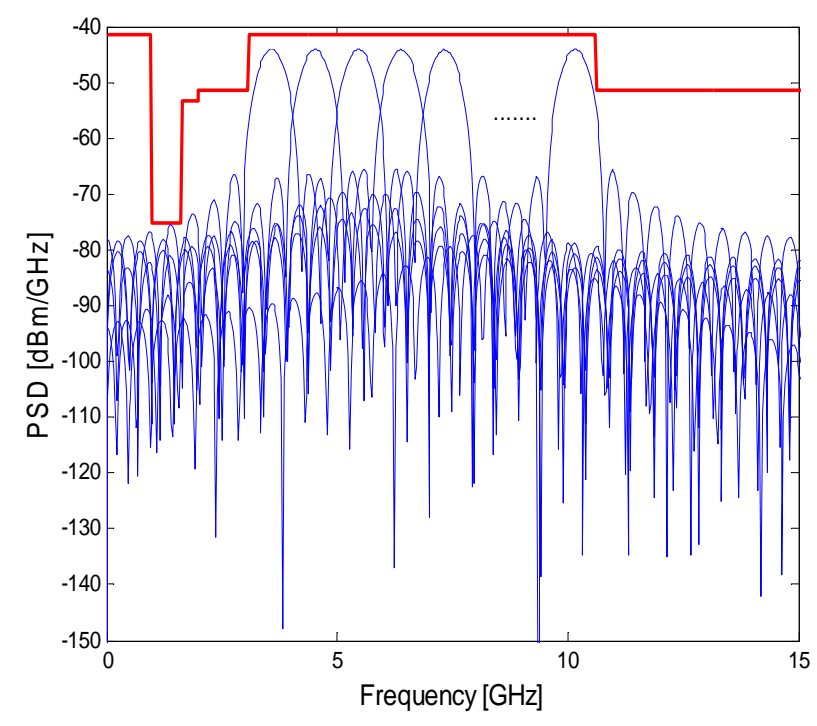

Figure 3. The multiband spectrum allocation diagram. 


\subsection{MB-IR-UWB System Model Building}

Consider a single-user MB-IR-UWB, with the binary orthogonal TH-PPM modulation, it is assumed that the pulse repetition time is $T_{s}$ and every $N_{s}$ pulses are used to transmit one bit information. So the binary symbol rate $R_{b}=1 / N_{s} T_{s}$ bps. The transmitted signal can be expressed as:

$$
\begin{aligned}
& S(t)=\sum_{i=0}^{N-1} s_{i}(t)=\sum_{i=0}^{N-1} \sum_{j=-\infty}^{+\infty} p_{i}\left(t-j T_{s}-c_{j} T_{c}-a_{j} \varepsilon\right) \\
& i=0,1, \cdots N-1
\end{aligned}
$$

where $s_{i}(t)$ is transmitted signal of the ith band, $p_{i}(t)$ is the pulse waveform of the $i$ th band, $c_{j} T_{c}$ defines the jitter of the pulse with respect to the timing of the integer, $a_{j} \varepsilon$ is the time shift caused by PPM modulation.

Trough AWGN channel, the received signal can be written as:

$$
r(t)=\alpha S(t-\tau)+n(t)
$$

Channel gain $\alpha$ and channel delay $\tau$ depends on the propagation distance between the transmitter and receiver. $n(t)$ is additive white Gaussian noise with zero mean value and variance $N_{0} / 2$.

As stated earlier, the pulses between each sub-band are orthogonal, the optimum receiver under the AWGN can be considered, the received signal goes into the correlators, multiplying correlation mask of each band $v_{i}(t)$, and into the integrator, then into the decision unit for judgment. The correlation mask of the ith band is

$$
v_{i}(t)=p_{i}\left(t-\tau-c_{j} T_{c}\right)-p_{i}\left(t-\tau-c_{j} T_{c}-\varepsilon\right)
$$

For each band is independent, separate judgments can be made in each branch. The detection is a standard hypothesis testing problem. While the decision variables

$$
Z_{i}=\int_{\tau}^{\tau+T_{s}} r(t) v_{i}(t) d t>0
$$

it will be judged bit ' 0 ', othervise bit ' 1 '. According to the receive form of binary determinate signal, usually in the case of equal priori probability, we can obtain the average error probability

$$
\begin{aligned}
P e & =\frac{1}{2} \operatorname{Prob}(Z>0 \mid Z<1)+\frac{1}{2} \operatorname{Prob}(Z<0 \mid Z>1) \\
& =\operatorname{Prob}(Z<0 \mid Z>1)
\end{aligned}
$$

Define $\rho=\frac{1}{E_{b R X}} \int_{0}^{T}\left[p_{0}(t) p_{1}(t)\right] d t$ is the normalized correlation coefficient of the received signal $p_{0}(t)$ and $p_{1}(t)$, where $E_{b R X}=\alpha^{2} N_{s} E_{T X}$, it's the energy of each received bit information. After some calculus operations, yield

$$
\begin{aligned}
P e & =\int_{\sqrt{\frac{E_{b R X}(1-\rho)}{N_{0}}}}^{+\infty} \frac{1}{\sqrt{2 \pi}} e^{-\frac{u^{2}}{2}} d u \\
& =\frac{1}{2} \operatorname{erfc}\left(\sqrt{\frac{E_{b R X}(1-\rho)}{2 N_{0}}}\right)
\end{aligned}
$$

For binary orthogonal PPM signal, we can easily obtain that

$$
\rho=\frac{1}{E_{b R X}} \int_{0}^{T} p_{0}(t) p_{0}(t-\tau) d t=0
$$

Thus, the average error probability of binary orthogonal PPM for MB-IR-UWB system is:

$$
P e=\frac{1}{2} \operatorname{erfc}\left(\sqrt{\frac{E_{b R X}}{2 N_{0}}}\right)
$$

where $\operatorname{erfc}(y)=\frac{2}{\sqrt{\pi}} \int_{y}^{+\infty} e^{-u^{2}} d u$.

\section{Simulations and Performance Analysis}

\subsection{Performance Comparison of Single-band and Multiband IR-UWB}

The following parts analyze MB-IR-UWB system performance from two views of reliability and validity.

First, for the reliability, Figure 4 shows the performance comparison of the single-band UWB and MB-IRUWB system, assumed the conditions of the same waveform, the same data transmission rate.

It can be seen from Figure 4 that the BER curves of MB-IR-UWB and single-band UWB almost completely overlap. This is because that each band can be equivalent to a single-band UWB, and in white Gaussian noise channel bit error rate is only related to signal-to-noise ratio. So in terms of reliability, multiband UWB system did not enjoy the advantage.

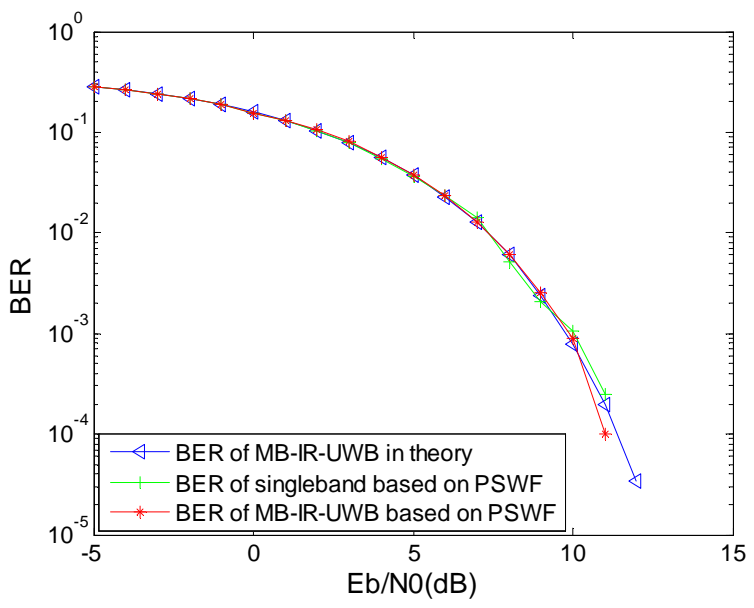

Figure 4. The performance comparison of multiband and single-band UWB. 
Second, for the validity, the transmission rate of the two systems is compared. When the operating frequency occupied the entire $7.5 \mathrm{GHz}$ band, the single-band PSWF pulse duration $T_{m 1}=4 n s$, multiband PSWF pulse duration $T_{m 2}=4 n s$. Therefore, if other conditions are the same, the pulse repetition period $T_{s 1}=T_{s 2}=N_{s} N_{h} T_{m 2}$. Suppose the transmission rate of single-band UWB is $R_{b 1}$, and the transmission rate of MB-IR-UWB is $R_{b 2}$, we can see that

$$
R_{b 1}=1 / N_{s} T_{s 1}, R_{b 2}=\left(1 / N_{s} T_{s 2}\right) \times N_{\text {sub }}
$$

Therefore, theoretically in equal time, compared with single-band UWB, the multi-band scheme can increase the transmission rate by $N$ times. Thus, multi-band UWB communications technology plays an important role in satisfying the increasingly high data transmission rate.

\subsection{The Impact of Pulse Duration on System Performance}

Due to the advantage of PSWF's high energy concentration, it is of great significance to increase the transmission rate as much as possible. From the analysis above, we can conclude that the pulse duration affects the data transmission rate indirectly. The following part will focus on the analysis of the effect of different $T_{m}$ on system performance. In the simulation, the number of pulse per bit $N_{s}=5$, the cardinality of the TH code $N_{h}=3$. The corresponding relation between different $T_{m}$ values and $R_{b}$ is shown as Table 1.

It can be seen from Figure 5 that the BER curves are very close when $T_{m}$ equals to $8 \mathrm{~ns}, 4 \mathrm{~ns}$, $2 \mathrm{~ns}$. Under this circumstance, the system performance is the best. When $T_{m}$ is $1.5 \mathrm{~ns}$, the system performance deteriorates seriously, and when $T_{m}$ is $1 \mathrm{~ns}$, the system performance becomes worst. It is because that the larger $T_{m}$ is, the higher energy concentration of pulse in particular frequency band is. So the less energy loss is, the better the system performance is. Thus, the validity and reliability is a pair of mutually contradictory unity. To improve the reliability of the system, it would inevitably lead to a decline in the validity. In our simulation, in order to ensure the system performance, the pulse duration which corresponds to $R_{b}=66.7 \mathrm{Mbps}$ is the best, i.e. $T_{m}=2$ ns.

\section{Conclusions}

This paper takes the MB-IR-UWB communication system

Table 1. Corresponding relation between different $T_{m}$ values and $\boldsymbol{R}_{\boldsymbol{b}}$.

\begin{tabular}{cccccc}
\hline Pulse duration $T_{m} / \mathrm{ns}$ & 8 & 4 & 2 & 1.5 & 1 \\
\hline Bit rate $R_{b} / \mathrm{Mbps}$ & 16.7 & 33.3 & 66.7 & 88.9 & 133.3 \\
\hline
\end{tabular}

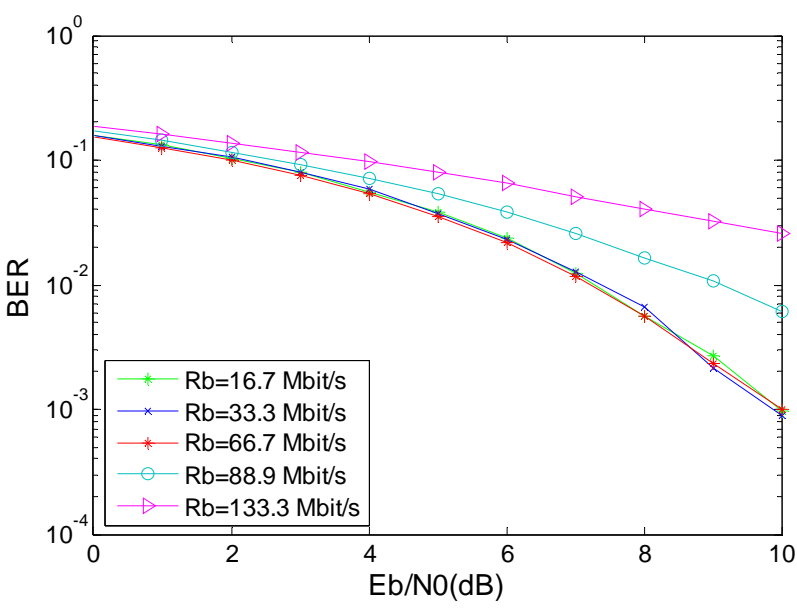

Figure 5. The performance comparison in different data rate.

based on PSWF pulse as the research object, proposes a new way of multi-band spectrum arrangement and uses coherent demodulation method to achieve the best reception. Through system model building and simulation analysis, we draw the conclusion that MB-IR- UWB system has the same bit error rate performance with single-band UWB. But in the MB-IR-UWB, the data can be transmitted in parallel, which can improve the transmission rate of information. In addition, PSWF pulse duration affects the BER performance. The simulation results show that the shorter PSWF pulse duration is, the larger the transmission rate is, but the system performance becomes worse. Therefore, in actual communication, we should make a tradeoff between the validity and reliability to a best choice.

\section{REFERENCES}

[1] L. Yang, Ultra-wideband Communications: An Idea Whose Time Has Come, Signal Processing Magazine, IEEE, Vol. 3, 2004, pp. 26-54. doi:10.1109/MSP.2004.1359140

[2] A. Batra et al., "Design of a Multiband OFDM System for Realistic UWB Channel Environments,” IEEE Transactions Microwave Theory and Techniqu, Vol. 52, No. 9, 2004, pp. 2123-2138. doi:10.1109/TMTT.2004.834184

[3] S. Paquelet, L.-M. Aubert and B. Uguen, "An Impulse Radio Asynchronous Transceiver for High Data Rates," International Workshop on Wideband Systems joint with Conference on Ultrawideband Systems and Technologies, Joint UWBST \& IWUWBS., pp. 18-21, 2004.

[4] Martin Mittelbach Rainer Moorfeld Adolf finger. Performance of a Multiband Impulse Radio UWB Achitecture. The 3rd Conference on mobile Technology, Applications and Systems 2006.

[5] B. Parr, B. Cho, K. Wallace and Z. Ding, "A Novel Ultra-Wideband Pulse Design Algorithm,” IEEE Communications Letters, No. 5, 2003, pp. 219-221. 
doi:10.1109/LCOMM.2003.812167

[6] Aamish Hasan Asim Anwar Hasan Mahmood, On the Perfomance of Multiband DS-UWB System for IEEE 802.15 a Channel with Hydrid PIC Rake Receiver. Proceedings-International Conference on Computer Networks and Information Technology, 2001, pp. 65-69.

[7] A. Hasan, A. Anwar and H. Machael, "on the Perfomance of Multiband DS-UWB System for IEEE 802.15a Channel with Hydrid PIC Rake Receiver. Proceedings-International Conference on Computer Networks and Information Technology, 2011, pp. 65-69.
doi:10.1109/ICCNIT.2011.6020909

[8] D. Slepian and H. O. Pollak, "Prolate Spheroidal Wave Functions, Fourier analysis, and Uncertainty-I,” Bell Syst. Tech. J., Vol. 40, No. 1, 1961, pp. 43-46. doi:10.1002/j.1538-7305.1961.tb03976.X

[9] W.-J. Hou and H. X. Wang, Multiband Orthogonal PSWF Pulses Used for M-ary PPM Ultra-Wideband System, International Conference on Instrumentation, Measurement, Computer, Communication and Control, 2011, pp. 589-592. 